\title{
The Mass of RL-NLS1 Black Holes: Reconciling Accretion Disk and Virial Estimates
}

\section{Giorgio Calderone*}

INAF-Osservatorio Astronomico di Trieste, Via G.B. Tiepolo 11, I-34143 Trieste, Italy

E-mail: giorgio.calderone@inaf.it

\section{Filippo D’Ammando}

INAF-Istituto di Radioastronomia, Via Gobetti 101, I-40129 Bologna, Italy

\section{Tullia Sbarrato}

Dip. di Fisica G. Occhialini, Università degli Studi di Milano-Bicocca, Piazza della Scienza 3, I-20126 Milano, Italy

\begin{abstract}
Radio-loud narrow-line Seyfert 1 galaxies (RL-NLS1) are characterized by relatively small widths of emission lines, hence their virial black hole mass estimates are typically smaller (and Eddington ratios correspondingly higher) than those of broad line Type 1 active galactic nuclei. However independent black hole mass estimation methods, such as those based on the accretion disk spectrum modeling, suggest that RL-NLS1 black hole masses are significantly larger than those estimated by virial methods, and that masses for both narrow and broad line Type 1 sources lie in the same range. In this paper we provide new black hole mass estimates for 25 RL-NLS1 sources (using the disk modeling method) and argue that a plausible way to solve the black hole mass discrepancy is to assume that the accretion disk in RL-NLS1 has a radiatively efficiency $\eta \sim 1 \%$, significantly lower than previously assumed.
\end{abstract}

Revisiting narrow-line Seyfert 1 galaxies and their place in the Universe - NLS1-2018

9-13 April 2018

Padova Botanical Garden, Italy

\footnotetext{
${ }^{*}$ Speaker.
} 


\section{Introduction}

Narrow-line Seyfert 1 Galaxies (NLS1, [1], [2], [3], [4], [5]) are a class of Type 1 active galactic nuclei (AGNs) characterized by small widths of the $\mathrm{H} \beta$ emission line $\left(\mathrm{FWHM} \lesssim 2000 \mathrm{~km} \mathrm{~s}^{-1}\right.$ ), a [O III] / $\mathrm{H} \beta$ luminosity ratio $<3$, and strong Fe II emission. The relatively small widths of Hydrogen lines implies that the mass of the accreting black holes is smaller than those typically observed in broad-line AGN and quasars (e.g. [6], [7], [8]) with values of $M_{\mathrm{BH}} \lesssim 10^{8} M_{\odot}$, as estimated using the single epoch virial method (e.g. [9], [10], [11]). Moreover these sources appear to lie below the $M-\sigma$ relation of inactive galaxies ([12], [7], [13]), suggesting that these objects may experience a different accretion process, or be in an early phase of their accretion history ([14]).

Despite the smaller black hole mass of NLS1 sources with respect to broad line AGN, 10\% of them show a significant emission at radio wavelengths (radio-loud NLS1, or RL-NLS1, [15]) and a few of them have a $\gamma$-ray detection ([16, 17], [18]), in some case with significant $\gamma$-ray variability $([19,20])$. Such "blazar-like" behaviour was once thought to be associated only with AGN with $M_{\mathrm{BH}} \gtrsim 10^{8} M_{\odot}$ (e.g. [21]), but eventually it became clear that RL-NLS1 sources are very similar to quasars ([22]), the only difference being their relatively lower luminosities and black hole masses. Another similarity between quasars and RL-NLS1 is that sources in both classes are likely powered by accretion onto a supermassive black hole, although possibly at different Eddington ratios. Given the observed luminosity of both quasars and RL-NLS1, the accretion process is likely mediated by a radiatively efficient accretion disk ([23]), possibly similar to an appropriately scaled Shakura \& Sunyaev disk ([24]). If this assumption is correct, the location and luminosity of the peak of the broadband spectrum at UV wavelengths provide important clues about the black hole mass and the accretion rate. Actually, this was historically the first method employed to estimate the black hole mass in AGN (e.g. [25], [26], [27]). However, interpreting the bulk of the emission at optical/UV wavelengths (the so-called "big blue bump") as an optically thick spectrum is definitely an oversimplification (e.g. [28]) and more complex models are required due to the relatively low temperature of the inner rings of the disk ([29]). Still, the Shakura \& Sunyaev model has been often used to model the broad-band energy distribution of broad-line AGN and quasars (e.g. [30], [31], [32], [33], [34]). In 2013, we used the accretion disk modeling method to analyze a sample of 17 RL-NLS1 ([35]) and find that our black hole mass estimates are significantly higher (a factor $\sim 6$ on average) than the corresponding virial mass estimates, and that the Eddington ratios were correspondingly lower. Hence, (at least) one of the two methods provide a biased mass estimate ${ }^{1}$

In this work we extend the analysis started in 2013 by considering new sources and a slightly modified mass estimation method ( $\$ 2)$, and discuss a plausible way to solve the RL-NLS1 black hole mass discrepancy $(\S 3)$.

\section{New black hole mass estimates}

For the new analysis we considered a sample of bona-fide RL-NLS1 [36], having an optical spectrum from SDSS, IR photometry from WISE and one or more Swift/UVOT observations. When available, we also included GALEX photometry. The optical spectra were required to estimate the

\footnotetext{
${ }^{1} \mathrm{~A}$ complete analysis of the assumptions and uncertainties in both methods is beyond the scope of this paper. See Calderone et al. 2013 ([35]) for a thorough discussion.
} 
continuum at optical/UV wavelength, disentangling the disk contribution from the host galaxy, Fe II emission and Balmer continuum, and measuring the emission-line widths. The analysis has been carried out using QSFIT ([37]), customized to accept both the SDSS data and the UV photometry, and to model the AGN continuum as an accretion disk spectrum (rather than a simple power law). The IR photometry were required to extrapolate the possible contribution from the synchrotron emission at optical/UV wavelengths. The UV photometry was required to constrain the luminosity and frequency of the peak of the accretion disk spectrum, in agreement with optical data. With these selection criteria we identified 31 sources with redshifts in the range $0.1<z<0.79$, but for 6 of them we could not identify the peak of the disk spectrum, either because of peculiar spectral shape or because the (non-simultaneous) observations at IR, optical and UV wavelengths were incompatible because of source variability. For the remaining 25 sources we obtained a reliable black hole mass and accretion rate estimate, although for 4 of these we only have an upper limit on the mass. An example of the analysis for the source PMN J0902+0442 $(z=0.53)$ is shown in Fig. 1. The orange line shows the QSFIT the accretion disk component. The luminosity and

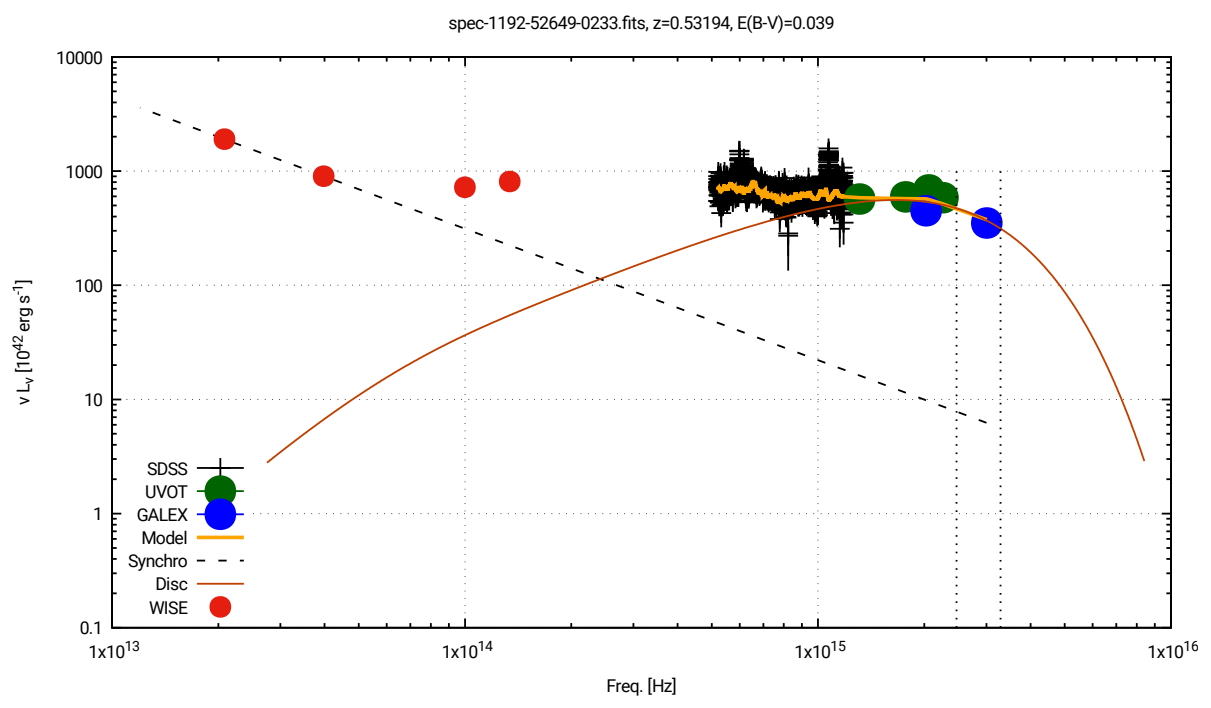

Figure 1: Analysis of PMN J0902+0442 $(z=0.53)$. The red, green and blue circles are respectively the WISE, GALEX and Swift/UVOT photometries (the error bars are smaller than the symbol). The black symbols are the SDSS data. The yellow solid line is the total QSFIT model, while the orange line is just the accretion disk component. The luminosity and frequency of the peak of the latter provides an estimate of the black hole mass and accretion rate (assuming a disk seen pole-on and a radiative efficiency $\sim 10 \%$ ). The synchrotron contribution (when non-negligible) is extrapolated from the IR photometry and subtracted from the optical/UV data before the fitting process.

frequency of the peak of such component provide an estimate of the black hole mass and accretion rate, assuming a disk seen pole-on and a radiative efficiency $\sim 10 \%$ (see [37] for a discussion on the reliability and impact of these hypothesis on the mass and accretion rate estimates). The synchrotron contribution (when non-negligible) is extrapolated from the IR photometry and subtracted from the optical/UV data before the fitting process. Beyond the black hole mass estimates obtained with the disk modeling method $\left(M_{\text {disc }}\right.$, to which we associated a very cautious uncertainty 
of 0.7 dex) we also calculated the single epoch virial mass ( $\left.M_{\mathrm{vir}, \mathrm{QSFit}}\right)$ using the FWHM of the H $\beta$ and $\mathrm{Mg}$ II lines and the continuum luminosity at $5100 \AA$ and $3000 \AA$ as estimated by QSFIT, and the calibration from [38]. Also, we collected the virial mass estimates from the literature ([15], [16], [39], [40], [8]). The results are shown in Fig. 2 (upper panel) where we used $M_{\text {vir,QSFit }}$ on the absicssa, and the ratio of the considered mass estimates over $M_{\mathrm{vir}, \mathrm{QSFit}}$ on the ordinate. The lower panel of Fig. 2 shows the relation between $M_{\text {disc }}$ and the radiative Eddington ratio (i.e., the bolometric disk luminosity divided by $1.3 \times 10^{38} M_{\text {disc }}$ ). As expected, all virial estimates cluster in
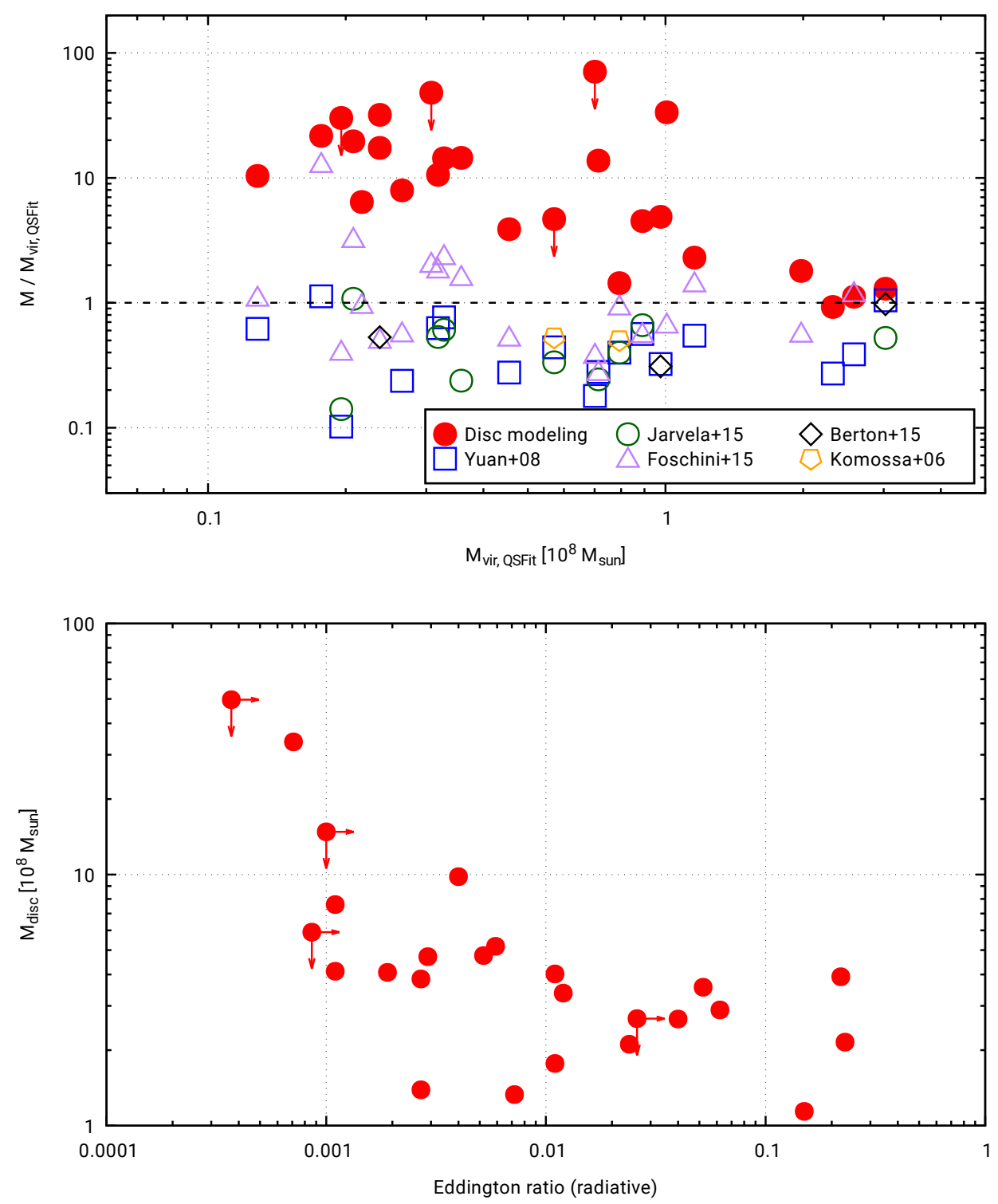

Figure 2: Upper panel: ratio of the several mass estimates over $M_{\mathrm{vir}, \mathrm{QSFit}}$, as a function of $M_{\mathrm{vir}, \mathrm{QSFit}}$. Lower panel: relation between $M_{\text {disc }}$ and the radiative Eddington ratio (i.e., the bolometric disk luminosity divided by $\left.1.3 \times 10^{38} M_{\text {disc }}\right)$.

a rather narrow, horizontal strip in the top panel. The slight offset of the virial mass estimates with 
respect to $M / M_{\text {vir,QSfit }} \sim 1$ is due to the updated calibration in [38] and references therein, which results in a factor $\sim 2$ higher mass estimates with respect to previous works. ${ }^{2}$ On the other hand, all disk modeling mass estimates are significantly larger than the virial masses by a factor of $\sim 10$ on average, and the Eddington ratios are correspondingly lower.

\section{Discussion and conclusions}

Even with the new mass estimates the RL-NLS1 black hole mass discrepancy discussed in $\S 1$ remains, and is even slightly exacerbated. Given the reliability of the single epoch virial mass method it is tempting to think that the disk modeling method should be somehow biased. However, there is a simple physical argument which shows that if the disk mass estimates are biased then the whole picture of an accretion disk emitting a multicolor, approximately thermal, spectrum (regardless of the temperature dependency with the radius) must be wrong. The argument is as follows: if the disk masses are overestimated, than the actual peak of the disk spectrum lies at higher frequencies with respect to what we found in our analysis. However, there is no physical process occurring in the neighborhood of the disk (e.g., in the atmosphere) able to shift the photons to lower frequency to produce the observed spectrum, since the black body emission already has the lowest temperature compatible with a given luminosity and emitting surface. The only way to lower the temperature (keeping a constant luminosity and radiative efficiency) is either to increase the area of the emitting surface of the disk (i.e., increase the mass of the black hole) or accept that the main engine of the RL-NLS1 is something different from a disk emitting a quasi-thermal spectrum at each annulus (see §6.1.1. of [35] for further discussion). Another plausible way to reconcile the disk modeling and virial estimates is to assume that the inner rings of the disk do not radiate efficiently, possibly because the energy is advected into the black hole before being radiated away. This occurs if the accretion rate is relatively high $(\gtrsim 1)$ and the disk undergoes a transition to a "slim" (rather than thin) disk ([42]). Note that in this case the total radiative efficiency would be much smaller than the commonly assumed value of $10 \%$. From the observational point of view the missing contribution from the inner rings is equivalent to a larger emitting surface, i.e., to a larger black hole mass as measured with the disk modeling method.

In summary, the only way to reconcile the disk modeling and virial mass estimates in the framework of the accretion disk theory is to assume that the total radiative efficiency of the disk must be of the order of 1\%, as shown in Fig. 2 (lower panel).

\section{Acknowledgements}

This conference has been organized with the support of the Department of Physics and Astronomy "Galileo Galilei", the University of Padova, the National Institute of Astrophysics INAF, the Padova Planetarium, and the RadioNet consortium. RadioNet has received funding from the European Union's Horizon 2020 research and innovation programme under grant agreement No 730562.

\section{References}

[1] D. E. Osterbrock and R. W. Pogge, The spectra of narrow-line Seyfert 1 galaxies, ApJ 297 (1985) 166.

\footnotetext{
${ }^{2}$ By using an older calibration for $M_{\mathrm{vir}, \mathrm{QSfit}}$, such as [41], all virial mass estimates would be consistent.
} 
[2] D. E. Osterbrock and R. W. Pogge, Optical spectra of narrow emission line Palomar-Green galaxies, ApJ 323 (1987) 108.

[3] R. W. Goodrich, Spectropolarimetry of 'narrow-line' Seyfert 1 galaxies, ApJ 342 (1989) 224.

[4] R. W. Pogge, Narrow-line Seyfert 1s: 15 years later, New Astronomy Review 44 (2000) 381.

[5] M.-P. Véron-Cetty, P. Véron and A. C. Gonçalves, A spectrophotometric atlas of Narrow-Line Seyfert 1 galaxies, A\&A 372 (2001) 730.

[6] B. M. Peterson, I. M. McHardy, B. J. Wilkes, P. Berlind, R. Bertram, M. Calkins et al., X-Ray and Optical Variability in NGC 4051 and the Nature of Narrow-Line Seyfert 1 Galaxies, ApJ 542 (2000) 161.

[7] H. Zhou, T. Wang, W. Yuan, H. Lu, X. Dong, J. Wang et al., A Comprehensive Study of 2000 Narrow Line Seyfert 1 Galaxies from the Sloan Digital Sky Survey. I. The Sample, ApJS 166 (2006) 128.

[8] E. Järvelä, A. Lähteenmäki and J. León-Tavares, Statistical multifrequency study of narrow-line Seyfert 1 galaxies, A\&A 573 (2015) A76.

[9] M. Vestergaard, Determining Central Black Hole Masses in Distant Active Galaxies, ApJ 571 (2002) 733.

[10] M. Vestergaard and B. M. Peterson, Determining Central Black Hole Masses in Distant Active Galaxies and Quasars. II. Improved Optical and UV Scaling Relationships, ApJ 641 (2006) 689.

[11] Y. Shen and X. Liu, Comparing Single-epoch Virial Black Hole Mass Estimators for Luminous Quasars, ApJ 753 (2012) 125.

[12] D. Grupe and S. Mathur, $M_{B H^{-}} \sigma$ Relation for a Complete Sample of Soft X-Ray-selected Active Galactic Nuclei, ApJ 606 (2004) L41.

[13] S. Komossa and D. Xu, Narrow-Line Seyfert 1 Galaxies and the M_BH- $\sigma$ Relation, ApJ 667 (2007) L33.

[14] S. Mathur, Narrow-line Seyfert 1 galaxies and the evolution of galaxies and active galaxies, MNRAS 314 (2000) L17.

[15] S. Komossa, W. Voges, D. Xu, S. Mathur, H. Adorf, G. Lemson et al., Radio-loud Narrow-Line Type 1 Quasars, AJ 132 (2006) 531.

[16] W. Yuan, H. Y. Zhou, S. Komossa, X. B. Dong, T. G. Wang, H. L. Lu et al., A Population of Radio-Loud Narrow-Line Seyfert 1 Galaxies with Blazar-Like Properties?, ApJ 685 (2008) 801.

[17] A. A. Abdo et al., Fermi/Large Area Telescope Discovery of Gamma-Ray Emission from a Relativistic Jet in the Narrow-Line Quasar PMN J0948+0022, ApJ 699 (2009) 976.

[18] F. D’Ammando, M. Orienti, J. Finke, C. M. Raiteri, E. Angelakis, L. Fuhrmann et al., SBS 0846+513: a new $\gamma$-ray-emitting narrow-line Seyfert 1 galaxy, MNRAS 426 (2012) 317.

[19] G. Calderone, L. Foschini, G. Ghisellini, M. Colpi, L. Maraschi, F. Tavecchio et al., $\gamma$-ray variability of radio-loud narrow-line Seyfert 1 galaxies, MNRAS 413 (2011) 2365.

[20] F. D’Ammando, M. Orienti, J. Finke, T. Hovatta, M. Giroletti, W. Max-Moerbeck et al., The awakening of the $\gamma$-ray narrow-line Seyfert 1 galaxy PKS 1502+036, MNRAS 463 (2016) 4469.

[21] M. Chiaberge and A. Marconi, On the origin of radio loudness in active galactic nuclei and its relationship with the properties of the central supermassive black hole, MNRAS 416 (2011) 917. 
[22] V. S. Paliya, M. Ajello, S. Rakshit, A. K. Mandal, C. S. Stalin, A. Kaur et al., Gamma-Ray-emitting Narrow-line Seyfert 1 Galaxies in the Sloan Digital Sky Survey, ApJ 853 (2018) L2.

[23] G. A. Shields, Thermal continuum from acretion disks in quasars, Nature 272 (1978) 706.

[24] N. I. Shakura and R. A. Sunyaev, Black holes in binary systems. Observational appearance., A\&A 24 (1973) 337.

[25] M. A. Malkan, The ultraviolet excess of luminous quasars. II - Evidence for massive accretion disks, ApJ 268 (1983) 582.

[26] W.-H. Sun and M. A. Malkan, Fitting improved accretion disk models to the multiwavelength continua of quasars and active galactic nuclei, ApJ 346 (1989) 68.

[27] W. Zheng et al., Astro-1 and ground-based observations of Markarian 335: Evidence for an accretion disk, ApJ 444 (1995) 632.

[28] A. Koratkar and O. Blaes, The Ultraviolet and Optical Continuum Emission in Active Galactic Nuclei: The Status of Accretion Disks, PASP 111 (1999) 1.

[29] C. Done, S. W. Davis, C. Jin, O. Blaes and M. Ward, Intrinsic disc emission and the soft X-ray excess in active galactic nuclei, MNRAS 420 (2012) 1848.

[30] G. Ghisellini and F. Tavecchio, Canonical high-power blazars, MNRAS 397 (2009) 985.

[31] D. M. Capellupo, H. Netzer, P. Lira, B. Trakhtenbrot and J. Mejía-Restrepo, Active galactic nuclei at z İC 1.5 - III. Accretion discs and black hole spin, MNRAS 460 (2016) 212.

[32] F. Shankar et al., The Optical-UV Emissivity of Quasars: Dependence on Black Hole Mass and Radio Loudness, ApJ 818 (2016) L1.

[33] B. Trakhtenbrot, M. Volonteri and P. Natarajan, On the Accretion Rates and Radiative Efficiencies of the Highest- redshift Quasars, ApJ 836 (2017) L1.

[34] J. E. Mejía-Restrepo, P. Lira, H. Netzer, B. Trakhtenbrot and D. M. Capellupo, The effect of nuclear gas distribution on the mass determination of supermassive black holes, Nature Astronomy 2 (2018) 63.

[35] G. Calderone, G. Ghisellini, M. Colpi and M. Dotti, Black hole mass estimate for a sample of radio-loud narrow-line Seyfert 1 galaxies, MNRAS 431 (2013) 210.

[36] F. D’Ammando, M. Orienti, J. Finke, J. Larsson, M. Giroletti and C. Raiteri, A Panchromatic View of Relativistic Jets in Narrow-Line Seyfert 1 Galaxies, Galaxies 4 (2016) 11.

[37] G. Calderone, L. Nicastro, G. Ghisellini, M. Dotti, T. Sbarrato, F. Shankar et al., QSFIT: automatic analysis of optical AGN spectra, MNRAS 472 (2017) 4051.

[38] J.-H. Woo et al., Calibration and Limitations of the Mg II Line-based Black Hole Masses, ApJ 859 (2018) 138.

[39] M. Berton, L. Foschini, S. Ciroi, V. Cracco, G. La Mura, M. L. Lister et al., Parent population of flat-spectrum radio-loud narrow-line Seyfert 1 galaxies, A\&A 578 (2015) A28.

[40] L. Foschini, M. Berton, A. Caccianiga, S. Ciroi, V. Cracco, B. M. Peterson et al., Properties of flat-spectrum radio-loud narrow-line Seyfert 1 galaxies, A\&A 575 (2015) A13.

[41] R. J. McLure and M. J. Jarvis, Measuring the black hole masses of high-redshift quasars, MNRAS 337 (2002) 109.

[42] M. A. Abramowicz, B. Czerny, J. P. Lasota and E. Szuszkiewicz, Slim accretion disks, ApJ 332 (1988) 646. 